\title{
Acknowledgement of manuscript reviewers, the underappreciated contributors
}

Yasmin Mulla, Ahmed Bakillah and M Mahmood Hussain*

\section{Contributing reviewers}

We and the Editorial Board sincerely acknowledge and thank all the reviewers for their active participation and contribution during 2014 (Volume 11). We greatly appreciate their dedication and behind the scenes contribution. It is largely due to their support and expertise that we have been able to publish high standard manuscripts. We would also like to thank authors for choosing Nutrition \& Metabolism and contributing their cherished work to this journal.

Sarah Abou Merhi

United States of America

Nader G. Abraham

United States of America

Charles Abramson

United States of America

Abdelaziz Abulelsaad

Egypt

Nihal Ahmad

United States of America

Kamal Amin

Egypt

Robin Anderson

United States of America

Susan Arthur

United States of America

Stella Aslibekyan

United States of America

Ramy Attia

United States of America

Maurizio Averna

Italy

Salman Azhar

United States of America
Ahmed Bakillah

United States of America

Michiel Balvers

Netherlands

Tyler Barker

United States of America

Darius Batulevicius

Lithuania

Jamie Baum

United States of America

Juerg H Beer

Switzerland

Elizabeth Beierle

United States of America

Damien Belobrajdic

Australia

Martha Belury

United States of America

\section{Melissa Benton}

United States of America

M'Hamed Bentourkia

Canada

Elizabeth Bertone-Johnson

United States of America
Deepak Bhatnagar
United Kingdom

Sheng Bi

United States of America

Jeffrey Billheimer

United States of America

William Blaner

United States of America

Emanuela Bostjancic

Slovenia

Olivier Bruyère

Belgium

Martin Burtscher

Austria

Andrew Butler

United States of America

Monika Cahova

Czech Republic

Edward Calabrese

United States of America

Philip Calder

United Kingdom

Matthew Cannon

United States of America

\footnotetext{
* Correspondence: Mahmood.Hussain@downstate.edu
}

Editor-in-Chief, Nutrition \& Metabolism 
Luciana Caperuto

Brazil

Annalisa Castagna

Italy

Rolando Ceddia

Canada

Lisa Ceglia

United States of America

Giovanna Cenacchi

Italy

Xueying Chen

United States of America

Dong Cheng

United States of America

Mi-La Cho

Korea, South

Robert Coker

United States of America

Ian Cook

South Africa

Emmanuel Cosson

France

Philippe Costet

United States of America

Giuseppe Crescenzi

Italy

Stephen Cunnane

Canada

Alexandre Da Silveira

Brazil

Dominic D'Agostino

United States of America

Kezhi Dai

United States of America

Mark Daly

United Kingdom

Erick De Oliveira

Brazil

Nicole De Wit

Netherlands

Tamás Decsi

Hungary

Hans Degens

United Kingdom
Christos Derdemezis

Greece

Latha Devi

United States of America

Wen-Xing Ding

United States of America

Mohamed Dkhil

Saudi Arabia

Lharbi Dridi

Canada

Slavik Dushenkov

United States of America

Michael Eades

United States of America

Peter Eck

Canada

Abdalla El-Mowafy

Egypt

Henk Everts

Netherlands

Richard Feinman

United States of America

Arny Ferrando

United States of America

Eduardo Ferriolli

Brazil

Yvonne Fierz

Switzerland

Carmelo Fiore

Italy

Pavel Flachs

Czech Republic

James Fleet

United States of America

Leng Huat Foo

Malaysia

Ju Yen Fu

Malaysia

Jose Fuentes

Spain

Mary C. Gannon

United States of America

Mingming Gao

United States of America
Diego Garcia-Diaz

Chile

Eduardo Garcia-Fuentes

Spain

Amalia Gastaldelli

Italy

Abhisek Ghosal

United States of America

Barbara Gower

United States of America

Olaf Grisk

Germany

Romain Harmancey

United States of America

Robert Harris

United States of America

Susan Harris

United States of America

Morey Haymond

United States of America

Gerwin Heller

Austria

Darren Henstridge

Australia

Chi-Tang Ho

United States of America

Conrad Hodgkinson

United States of America

Adenilda Honorio-França

Brazil

Mary Honors

United States of America

Philip Hooper

United States of America

Chao-Tien Hsu

Taiwan

Yu Huang

China

Juha Hulmi

Finland

Jahangir Iqbal

United States of America

Eui-Bae Jeung

Korea, South 
Xian-Cheng Jiang

United States of America

Mirim Jin

Korea, South

Julie Jurenka

United States of America

Douglas Kalman

United States of America

Konstantin Kandror

United States of America

Tadashi Kawai

Japan

Daniel Konrad

Switzerland

Mark Kotowicz

Australia

Chao-Qiang Lai

United States of America

Marcia Latorraca

Brazil

Donald Layman

United States of America

Mélanie Lemire

Canada

Tung Ming Leung

United States of America

Nate Lewis

United States of America

Ke Li

United States of America

Rui Li

United States of America

Qiang Li

United States of America

Fabio Lima

Brazil

Pao-Hwa Lin

United States of America

I-Min Liu

Taiwan

George Liu

China

Kiatlyn Liu

United States of America
Agnieszka Loboda

Poland

Jose Lopez-Miranda

Spain

Emil Lou

United States of America

Guojun Lu

United States of America

Bing Luan

United States of America

Marco Manca

Netherlands

Gaowei Mao

United States of America

Maria Raquel Marçal Natali

Brazil

\section{Carmen Martinez}

France

Vera Mazurak

Canada

Bodo Melnik

Germany

Ali Metwalli

Saudi Arabia

Christine Miaskowski

United States of America

Luca Miele

Italy

Mohammad Minhajuddin

United States of America

Bettina Mittendorfer

United States of America

Mahmoud Mohammad

United States of America

Srinidi Mohan

United States of America

Lesley Moisey

Canada

Maria Murholm

Denmark

Elza Muscelli

Brazil

Thirupathi Muthusamy

United States of America
Fernando Naclerio

United Kingdom

Takafumu Nagatomo

Japan

Joseph Nickels

United States of America

Estela Maria Novak

Brazil

Toshikatsu Okumura

Japan

Rytas Ostrauskas

Lithuania

Alok Pachori

United States of America

Xiaoyue Pan

United States of America

Athanasios G. Papavassiliou

Greece

Sandra Peake

Australia

Michael Pearen

Australia

Sandra Peters

Canada

Stephen Phinney

United States of America

Hanno Pijl

Netherlands

Earl Prinsloo

South Africa

Spencer Proctor

Canada

Denis Prud'Homme

Canada

Radhakrishna Rao

United States of America

Angela Resende

Brazil

Jong Rho

Canada

Denis Richard

Canada

Gerald Rimbach

Germany 


Roberto Rivabene
Italy
Catarina Rodrigues Dos Santos
Portugal
Jean-Max Rouanet
France
Helene Rundqvist
Sweden
Arkady Rutkovskiy
Norway
Jennifer Ryan
United Kingdom

Fermin Sanchez De Medina Spain

Sushma Sharma

United States of America

Fareeba Sheedfar

Netherlands

Laura Shelton

United States of America

Sangeetha Shyam

Malaysia

Adrian Slee

United Kingdom

James Soh

United States of America

Evelien Sohl

Netherlands

Guisheng Song

United States of America

Carol Souza Da Silva

Netherlands

Shanthi Srinivasan

United States of America

Alan Steel
United Kingdom
Barbara Strasser
Austria
Anne Sumner
United States of America
Guang Sun
Canada

\author{
Cenk Suphioglu \\ Australia
}

Arthur Swislocki

United States of America

Toshiaki Tamaki

Japan

Daniel Teitelbaum

United States of America

Inge Tetens

Denmark

Marie Thearle

United States of America

Min Tian

United States of America

Uwe Tietge

Netherlands

Angela Ting

United States of America

Janet Tou

United States of America

Robbert Touwslager

Netherlands

Ulrike Trendelenburg

United Kingdom

Tsuyoshi Tsuduki

Japan

Mariko Uehara

Japan

Angela Valverde

Spain

Evert Van Schothorst

Netherlands

Vasundara Venkateswaran

Canada

Adriana C Vidal

United States of America

Meghan Walsh

United States of America

Wan Zurinah Wan Ngah

Malaysia

Doug Ward

United Kingdom
Hope Weiler

Canada

Klaas Westerterp

Netherlands

Margriet Westerterp-Plantenga

Netherlands

Wanwarang Wongcharoen

Thailand

Zengying Wu

United States of America

Min Xia

China

Xunde Xian

United States of America

Feifei Xiao

United States of America

Yuquan Xiong

United States of America

Kazumasa Yamagishi

United States of America

Hua Yang

China

Kaiping Yang

Canada

Qinglin Yang

China

Zhong Ye

United States of America

Masanori Yoshizumi

Japan

Kai Yuan

United States of America

Fouad Zakharia

United States of America

Xiaodong Zhang

United States of America

Jinzhong Zhang

United States of America

Received: 21 January 2015 Accepted: 21 January 2015

Published: 4 February 2015

doi:10.1186/1743-7075-12-3

Cite this article as: Mulla et al::

Acknowledgement of manuscript reviewers,

the underappreciated contributors. Nutrition

\& Metabolism 2015 12:3. 